DOI:

УДК 621.785.5: 621.793.6

Б.П. Середа, д.т.н., проф., seredabp@ukr.net

О.С. Гайдаєнко, аспірант, seredabp@ukr.net

Д.Б. Середа, к.т.н., ст. викладач, seredabp@ukr.net

Дніпровський державний технічний університет, м. Кам'янське

\title{
ТЕРМОДИНАМІЧНЕ МОДЕЛЮВАННЯ ОТРИМАННЯ АЛІТОВАНИХ ПОКРИТТІВ ЛЕГОВАНИХ ТИТАНОМ В УМОВАХ САМОРОЗПОВСЮДЖУВАЛЬНОГО ВИСОКОТЕМПЕРАТУРНОГО СИНТЕЗУ
}

\begin{abstract}
В роботі розглянуто термодинамічне моделювання отримання алітованих покриттів легованих титаном в умовах саморозповсюджувального високотемпературного синтезу. Дослідження механізму формування захисного покриття на конструкиійних матеріалах здійснювали, використовуючи метод термодинамічного аналізу можливих хімічних реакцій між компонентами системи. Для иього був виконаний розрахунок взаємодії газового насичуваного середовища, що утворюється в процесі СВС і матеріалом. Для термодинамічного аналізу $C B C-$ проиесу формування алітованих покриттів викорисовується універсальна програма розрахунку багатокомпонентних гетерогенних систем TERRA, створена на основі програми ACTPA-4 під середовище WINDOWS і відпрачьована для високотемпературних прочесів. На відміну від традиційних у хімічній термодинаміці методів розрахунків параметрів рівноваги з використанням енергії Гиббса, констант рівноваги та закону діючих мас Гольдберга і Ваге, універсальна програма термодинамічних розрахунків TERRA, базується на принципі максимуму ентропії для ізольованих термодинамічних систем, що перебувають у стані рівноваги. Воно характеризується максимумом ентропії щодо термодинамічних ступенів свободи, до яких відносяться концентрації компонентів системи, температура, тиск.
\end{abstract}

Ключові слова: моделювання; термодинаміка; синтез; покриття; алюміній; титан.

The paperr considers thermodynamic modeling of obtaining titanium-doped aluminized coatings under conditions of self-temperature high temperature synthesis. Following the mechanism for the formation of a protective coating on structural materials, the method of thermodynamic analysis of possible chemical reactions between the components of the system was followed. For this purpose, the calculation of the interaction of the gas saturable medium formed in the process of SHS and material was performed. For the thermodynamic analysis of the SHS process for the formation of aluminized coatings, a universal program for calculating multicomponent heterogeneous TERRA systems, created on the basis of the ASTRA-4 program for the WINDOWS environment, has been developed for high-temperature processes. Unlike traditional methods in chemical thermodynamics for calculating equilibrium parameters using Gibbs energy, equilibrium constants and the law of effective mass of Goldberg and Wage, the universal program of thermodynamic calculations TERRA is based on the principle of maximum entropy for isolated thermodynamic systems in equilibrium. It is characterized by the maximum entropy in thermodynamic degrees of freedom, which include the concentration of system components, temperature, pressure.

Keywords: modeling; thermodynamics; synthesis; coatings; aluminum; titanium.

\section{Постановка проблеми}

Дослідження механізму формування захисного покриття на конструкційних матеріалах здійснювали, використовуючи метод термодинамічного аналізу можливих хімічних реакцій між компонентами системи. Для цього був виконаний розрахунок взаємодії газового насичуваного середовища, що утворюється в процесі СВС і матеріалом. Для термодинамічного аналізу CBC-процесу формування алітованих покриттів використовується універсальна програма розрахунку багатокомпонентних гетерогенних систем TERRA, створена на основі програми ACTPA-4 [1-5] під середовище WINDOWS і відпрацьована для високотемпературних процесів. На відміну від традиційних у хімічній термодинаміці методів розрахунків параметрів рів- 
новаги з використанням енергії Гиббса, констант рівноваги та закону діючих мас Гольдберга i Ваге, універсальна програма термодинамічних розрахунків TERRA, базується на принципі максимуму ентропії для ізольованих термодинамічних систем, що перебувають у стані рівноваги. Воно характеризується максимумом ентропії щодо термодинамічних ступенів свободи, до яких відносяться концентрації компонентів системи, температура, тиск.

Аналіз останніх досліджень та публікацій

При протіканні однієї СВС-реакції термодинамічний розрахунок [6] величини адіабатичної температури взаємодії $T_{a}$ грунтуються на наступному допущенні [7]: реакція протікає повністю (зі спутенем перетворення $100 \%$ в в адіабатичних умовах, тобто теплота реакції при початковій температурі $T_{0}\left(T_{0}>298 \mathrm{~K}\right)$ витрачається на розігрівання до $T_{a d}$ і можливе плавлення продуктів. Розрахунок зводиться до визначення величини $T_{a}$ з нелінійного рівняння балансу ентальпії:

$$
\begin{gathered}
\Delta H_{T_{0}}(r)=\sum_{r}\left[\Delta H_{298}^{0}(r)+\int_{298}^{T_{0}} c_{p}(r) d T\right]=\Delta H_{T_{a d}}(p r)= \\
=\sum_{r}\left[\Delta H_{298}^{0}(p r)+\left(1-x_{m}\right) \int_{298}^{T_{a d}} c_{p}\left(p r_{s}\right) d T+x_{m}\left(\int_{298}^{T_{m}} c_{p}\left(p r_{m}\right) d T+\Delta H_{m}\left(p r_{m}\right)+\int_{T_{m}}^{T_{a d}} c_{p}\left(p r_{m}\right) d T\right],\right.
\end{gathered}
$$

де $\Delta H_{298}^{0}$ - стандартна ентальпія утворення речовини (для елементів $\Delta H_{298}^{0}=0$ ); $\Delta H_{m}$ и $T_{m}$ - теплота і температура плавлення; $c_{p}-$ теплоємність; $x_{m}-$ мольна частка розплаву в продуктах реакції; 2 - реагент; $p r_{m}$ - продукти реакції; нижні індекси s i ш відносяться до твердих речовин і розплаву відповідно.

Як видно $з$ формули (1), величина $T_{a d}$ визначається різницею між початковою ентальпією системи і їі ентальпією в рівноважному стані при $T_{a d}$. Проте такий підхід вірний тільки для малого числа реагентів і можливих реакцій і не підходить для багатокомпонентних гетерогенних систем, в яких можливе одночасне протікання декількох конкуруючих реакцій з утворенням різних продуктів. Крім того, у формулі (1) не враховується можливість неповного перетворення: як відомо, жодна реакція не протікає повністю - завжди встановлюється рівновага між продуктами і реагентами при деякій концентрації і тих і інших.

Тому для багатофазних багатокомпонентних систем необхідно застосовувати інші методи розрахунку термодинамічної рівноваги. Останніми роками для ТМ використовують комп'ютерні програми з термодинамічними базами даних (ТБД), які грунтуються на пошуку мінімуму енергії Гіббса багатофазної багатокомпонентної системи $[8,9,10-13]$. При цьому ТМ зводиться до пошуку в ТБД з'єднань, які можуть утворитися з елементів, що входять до складу початкових речовин, і визначенню їх кількісного співвідношення, що відповідає рівновазі за заданих умов. Для CBC-процесів у багатокомпонентних системах в Інституті структурної макрокінетики і проблем матеріалознавства РАН (ICMAН, м. Чорноголівка Московської обл.) розроблена спеціалізована програма ICMАН-ТЕРМО, яка дозволяє проводити ТМ тільки в адіабатичному режимі $[10,11]$. Розроблена в МГТУ ім. Н.Е. Баумана універсальна програма АСТРА-4 виконує пошук рівноважного складу, що відповідає умові локального максимуму ентропії системи $\mathrm{S}$ за наявності обмежень (умова збереження маси кожного елементу і повної внутрішньої енергії системи) для заданого початкового складу і термодинамічного режиму $[12,13]$. ТБД програми АСТРА-4 грунтована на довідковій літературі по термодинаміці індивідуальних речовин [14-18] і включає велике число неорганічних з'єднань.

\section{Формулювання мети дослідження}

Метою роботи є отримання рівномасового складу продуктів системи для алітування при тепловому самозайманні в умовах саморозповсюджувального високотемпературного синтезу. 


\section{Виклад основного матеріалу}

Порошкові насичуючі шихти при алітуванні в умовах СВС [19-20] мають у своєму складі хромисту складову, алюміній, газотранспортні активатори, а також титан, хром та кремній.

Порівняльна термодинамічна оцінка насичення міді газовим і парогазовим методом, зумовлює доцільність застосування одного з цих методів насичення міді і ії сплавів кремнієм, алюмінієм, титаном, хромом і нікелем.

Рівноважний склад СВС-шихт в режимі теплового самозаймання розраховувався для систем:

$$
\begin{aligned}
& \mathrm{XC}+\mathrm{Al}+\mathrm{Si}+\mathrm{Al}_{2} \mathrm{O}_{3}+\mathrm{NH}_{4} \mathrm{I}+\mathrm{AlF}_{3}, \\
& \mathrm{XC}+\mathrm{Al}+\mathrm{Ti}+\mathrm{Al}_{2} \mathrm{O}_{3}+\mathrm{NH}_{4} \mathrm{I}+\mathrm{AlF}_{3}, \\
& \mathrm{XC}+\mathrm{Al}+\mathrm{Cr}+\mathrm{Al}_{2} \mathrm{O}_{3}+\mathrm{NH}_{4} \mathrm{I}+\mathrm{NH}_{4} \mathrm{~F} .
\end{aligned}
$$

Для великого діапазону зміни вихідних компонентів в залежності від температури.

В результаті термодинамічної розрахунку рівномасового складу продуктів реакції можна запропонувати наступну кінетичну схему хімічних перетворень в досліджуваних системах:

1. Реакція горіння $(\mathrm{XC})$ :

$$
\mathrm{Cr}_{2} \mathrm{O}_{3}+2 \mathrm{Al}=2 \mathrm{Cr}+\mathrm{Al}_{2} \mathrm{O}_{3} .
$$

2. Реакції розпаду газотранспортного носія:

$$
\begin{aligned}
& \mathrm{AlF}_{3} \rightarrow \mathrm{Al}+3 \mathrm{~F}, \\
& \mathrm{NH}_{4} \mathrm{I} \rightarrow \mathrm{NH}_{3}+\mathrm{HI}, \\
& \mathrm{H} \mathrm{I} \rightarrow \mathrm{H}+\mathrm{I}, \\
& 2 \mathrm{NH}_{3} \rightarrow \mathrm{N}_{2}+3 \mathrm{H}_{2}, \\
& \mathrm{NH}_{4} \mathrm{~F} \rightarrow \mathrm{NH}_{3}+\mathrm{H} \mathrm{F}, \\
& \mathrm{HF} \rightarrow \mathrm{H}+\mathrm{F} .
\end{aligned}
$$

3. Хімічні транспортні реакції:

$$
\begin{aligned}
& \mathrm{Cr}+2 \mathrm{~F}=\mathrm{CrF}_{2}, \\
& \mathrm{Cr}+\mathrm{F}_{2}=\mathrm{CrF}_{3}, \\
& \mathrm{CrF}_{2}+1 / 2 \mathrm{~F}_{2}=\mathrm{CrF}_{3}, \\
& \mathrm{Al}+3 / 2 \mathrm{Cl}_{3}=\mathrm{AlCl}_{3}, \\
& \mathrm{Al}+\mathrm{I} \leftrightarrow \mathrm{AlI}, \\
& 2 \mathrm{Cr}+3 \mathrm{Cl}_{2}=2 \mathrm{CrCl}_{3}(2), \\
& \mathrm{Ti}+4 \mathrm{I}=\mathrm{TiI}_{4}, \\
& \mathrm{Ti}+3 \mathrm{~F} \leftrightarrow \mathrm{TiF}_{3}, \\
& \mathrm{Cr}+\mathrm{Cl}_{2} \leftrightarrow \mathrm{CrCl}_{2(\mathrm{r})}, \\
& \mathrm{Cr}+\mathrm{Cl}_{2} \leftrightarrow \mathrm{CrCl}_{2(ж)}, \\
& 2 \mathrm{Al}+3 \mathrm{I}_{2}=\mathrm{Al}_{2} \mathrm{I}_{6}, \\
& \mathrm{Al}+3 \mathrm{I}^{\leftrightarrow} \leftrightarrow \mathrm{AlI}_{3} .
\end{aligned}
$$

В результаті горіння порошкових сумішей з ГТА можливе утворення газової фази, яка містить сполуки $\mathrm{I}_{2}, \mathrm{Cl}, \mathrm{F}$ з вхідними в неї хімічними елементами. Зі збільшенням температури спостерігається збільшення кількості галогенідів (рис. 1,2). Це підтверджує можливість перенесення легуючих елементів для утворення покриттів.

Зі збільшенням температури кількість продуктів в газоподібній фазі зростає і виділяються конденсовані продукти. Характерно, що в області температур 700-1600 К частка конденсованої фази падає, що пов'язано з випаровуванням носія. Одночасно, починаючи з температури 800 К, відбувається розпад продуктів реакції, що підтверджує поява продуктів розкладання і різке збільшення кількості молей газу. Газоподібні продукти, взаємодіють з елементами порошкової системи переводять в газову фазу. При температурах вище 800 К частка конденсованої фази практично не змінюється. Цей факт дає підставу припустити, що в температурному інтервалі 700-1600 К відбуваються реакції з виділенням конденсованої фази, але без зміни числа молей, що характерно для реакцій розпаду, обміну з підкладкою, тобто по суті відбувається хімічний транспорт елементів. 


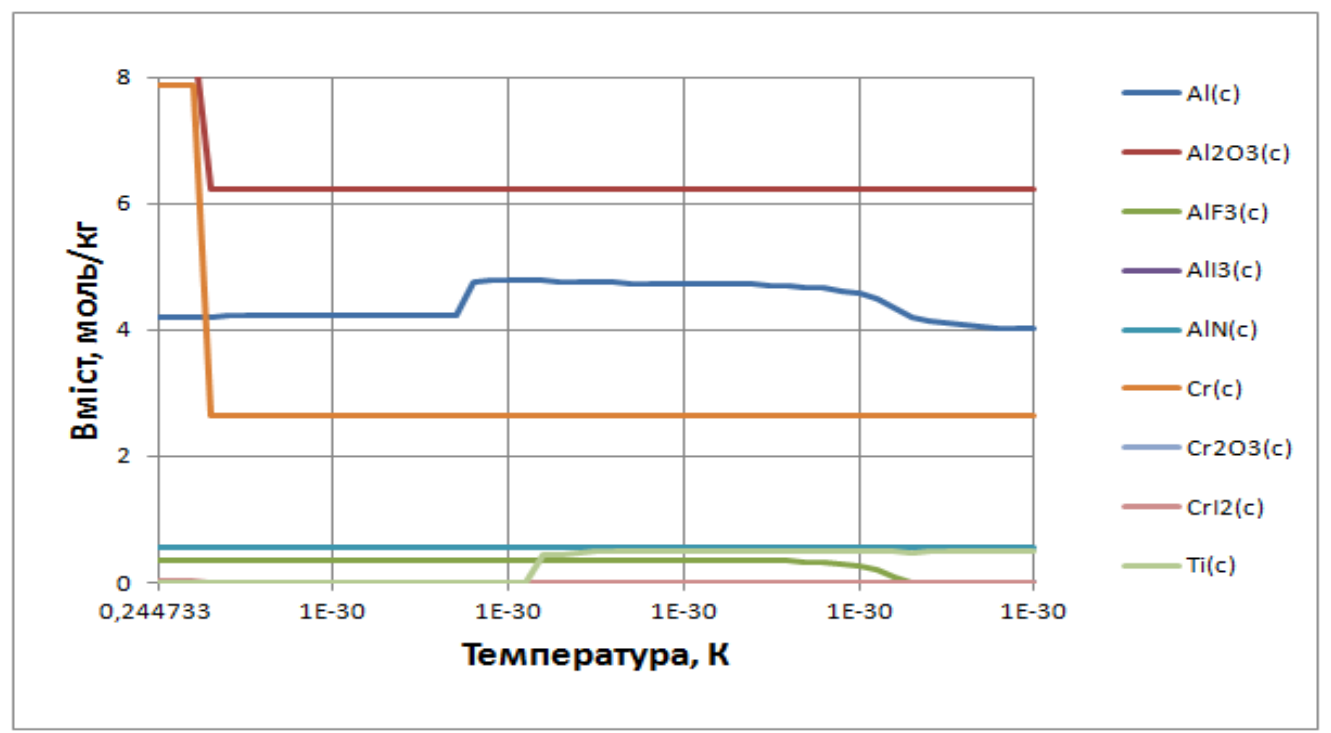

Рuc. 1. Вміст конденсованих продуктів в реакторі в режимі теплового самозаймання СВС-шихти для системи: $25 \% \mathrm{XC}+15 \% \mathrm{Al}+15 \% \mathrm{Ti}+40 \% \mathrm{Al}_{2} \mathrm{O}_{3}+2 \% \mathrm{NH}_{4} \mathrm{I}+3 \mathrm{AlF}_{3}$

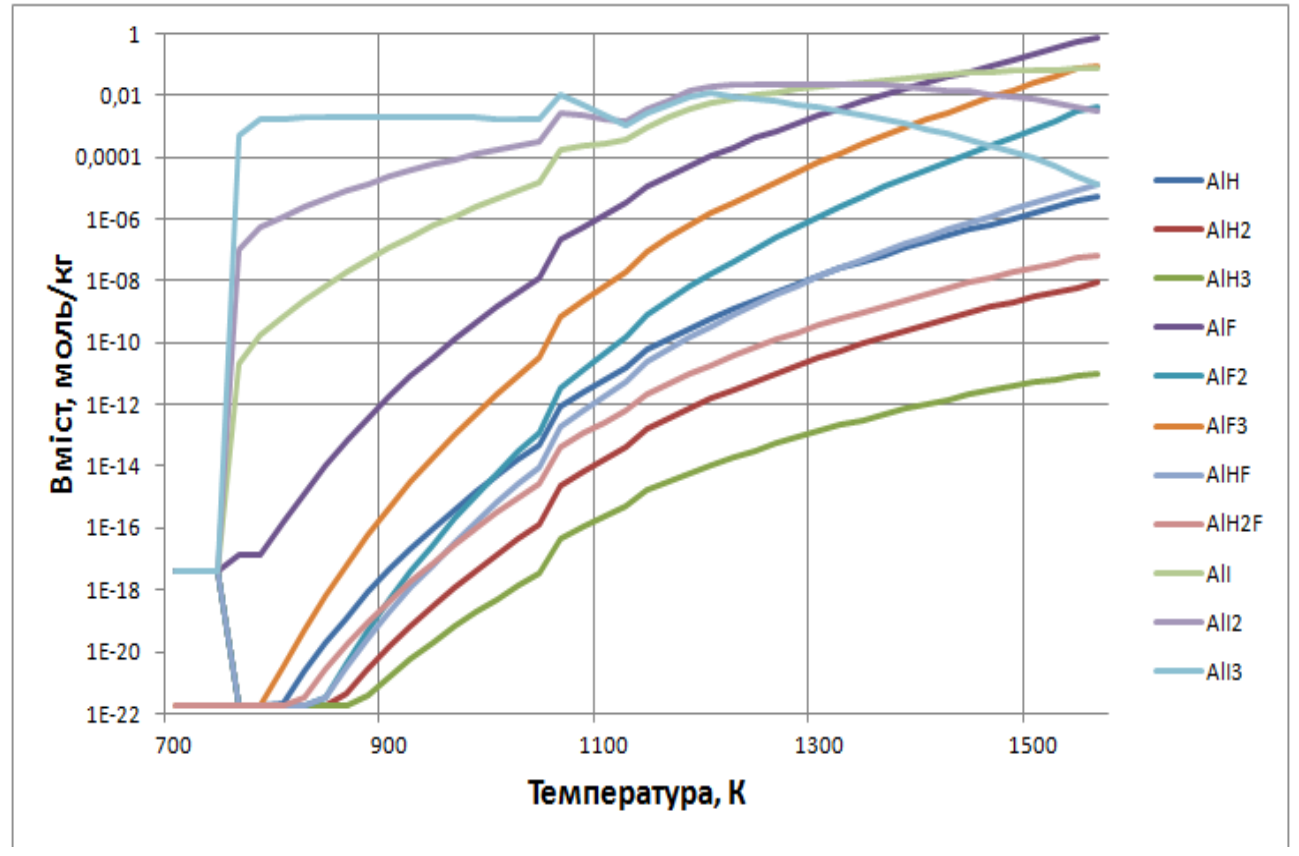

Рuc. 2. Вміст газоподібних сполук алюмінію в реакторі в режимі теплового самозаймання СВС-шихти для системи: $25 \% \mathrm{XC}+15 \% \mathrm{Al}+15 \% \mathrm{Ti}+40 \% \mathrm{Al}_{2} \mathrm{O}_{3}+2 \% \mathrm{NH}_{4} \mathrm{I}+3 \mathrm{AlF}_{3}$

Вміст газоподібних сполук алюмінію в реакторі в режимі теплового самозаймання СВСшихти для системи: $25 \% \mathrm{XC}+15 \% \mathrm{Al}+15 \% \mathrm{Ti}+40 \% \mathrm{Al}_{2} \mathrm{O}_{3}+2 \% \mathrm{NH}_{4} \mathrm{I}+3 \mathrm{AlF}_{3}$.

В діапазоні температур 700-1600 K є: $\mathrm{AlH}, \mathrm{AlH}_{2}, \mathrm{AlH}_{3}, \mathrm{AlF}, \mathrm{AlF}_{2}, \mathrm{AlF}_{3}, \mathrm{AlHF}, \mathrm{AlH}_{2} \mathrm{~F}$, AlI, $\mathrm{AlI}_{2}, \mathrm{AlI}_{3}$. Вміст газоподібних сполук хрому: $\mathrm{Al}(\mathrm{c}), \mathrm{Al}_{2} \mathrm{O}_{3}(\mathrm{c}), \mathrm{AlF}_{3}(\mathrm{c}), \mathrm{AlI}_{3}(\mathrm{c}), \mathrm{AlN}(\mathrm{c}), \mathrm{Cr}(\mathrm{c})$, $\mathrm{Cr}_{2}(\mathrm{c}), \mathrm{CrI}(\mathrm{c}), \mathrm{Ti}(\mathrm{c})$. Вміст газоподібних сполук титану: $\mathrm{TiOH}, \mathrm{TiF}, \mathrm{TiF}_{2}, \mathrm{TiF}_{3}, \mathrm{TiF}_{4}, \mathrm{TiOF}_{2}, \mathrm{TiI}$, $\mathrm{TiI}_{2}, \mathrm{TiI}_{3}, \mathrm{TiI}_{4}, \mathrm{TiOF}$. А також вміст конденсованих продуктів: $\mathrm{Al}(\mathrm{c}), \mathrm{Al}_{2} \mathrm{O}_{3}(\mathrm{c}), \mathrm{AlF}_{3}(\mathrm{c}), \mathrm{AlI}_{3}(\mathrm{c})$, $\operatorname{AlN}(\mathrm{c}), \mathrm{Cr}(\mathrm{c}), \mathrm{Cr}_{2}(\mathrm{c}), \mathrm{CrI}(\mathrm{c}), \mathrm{Ti}(\mathrm{c})$. 
В результаті проведеного дослідження отримані леговані алітовані покриття, що наведені на рис. 3.
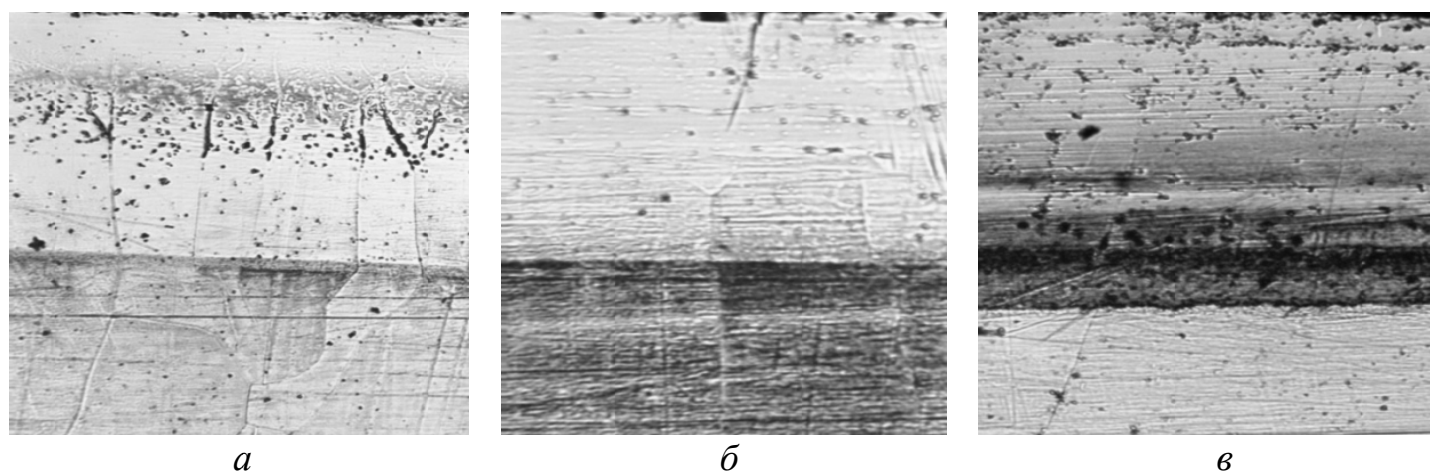

Puc. 3. Мікроструктури легованих агітованих покриттів в залежності від виду легування, на технічному залізі отриманих при $t_{\text {п }}-1000{ }^{\circ} \mathrm{C}, \tau_{\mathrm{B}}=60$ хв.: $a-\mathrm{Cr}(\times 100) ; \sigma-\mathrm{Ti}(\times 100)$; в- $\mathrm{Si}(\times 100)$.

\section{Висновки та перспективи подальших досліджень}

В результаті термодинамічного розрахунку рівномасового складу продуктів реакції отримано кінематичні схеми хімічних перетворень у досліджуваних системах. Визначено конденсовані та газоподібні сполуки. Доведено, що в температурному інтервалі 700-1600 К відбуваються реакції з виділенням конденсованої фази, але без зміни числа молей, що характерно для реакцій розпаду, обміну з конструкційним матеріалом, тобто відбувається хімічний транспорт елементів.

\section{Список використаної літератури}

1. Термодинамические свойства индивидуальных веществ: Справочное издание в 4-х т. / Л. В. Гурвич, И. В. Вейц, В. А. Медведев и др. - М: Наука, 1978.

2. JANAF Thermochemical Tables (Third Edition). J.Phys. Chem. Ref. Data vol.14 (1985) Suppl. No.1. (см. также NIST Chemistry WebBook: http://webbook.nist.gov).

3. Едильбаев А.И. / Исследование возможности обезжелезивания марганцевых концентратов / Вестник Национальной инженерной академии РК Алматы., 2008. - № 4. - С. 125-133.

4. Применение ЭВМ для термодинамических расчетов металлургических процессов / [ Синярев Г. Б., Ватолин Н. А.,Трусов Б. Г., Моисеев Р. К. и др.]. - М.: Наука, 1982. - 263 с.

5. Змий В.И. Особенности вакуумного активированного диффузионного насыщения металлов: термодинамика, механизм и кинетика /В.И. Змий, С.Г. Руденький // Металлофизика и новейшие технологии., 1998 - № 10. - С. 69-75.

6. Ловшенко Ф. Г. Термодинамическое моделирование гетерогенного взаимодействия при механическом легировании в системах на основе меди / Ф.Г Ловшенко, Г.Ф. Ловшенко, Б. Б. Хина, 3. М. Ловшенко, И. А. Лозиков //Вестник Белорусско-Российского университета., 2012. -№ 1(34). - С. 23-35.

7. Применение ЭВМ для термодинамических расчетов металлургических процессов / Г. Б. Синя-р в [и др.]. - М.: Наука, 1982. - 264 с.

8. Mason, P. Modeling thermodynamic processes / P. Mason, M. Mignanelli // Advanced Materialsand Processes., 1998. - Vol. 153, №. 4. - P. 21-24.

9. Shiryaev, A. Thermodynamics of SIIS processes: An advanced approach / A. Shiryaev // International Journal of SHS., 1995. - № 4 . - P. 351-362.

10. Barin, I. Thermoeheinical Properties of Inorganic Substances. Supplement / I. Barin, O. Knacke, 0. Kubaschevski. - Berlin: Springer-Verlag, 1977. - 243 p. 
11. Термодинамические свойства неорганических, веществ : справочник / У. Д. Верятин [и др.]. - Атомиздат, 1965. - 460 с.

12. Термические константы веществ / Под ред. В.ІІ. Глушко М. ВИНИТИ, 1979-197 с.

13. Термодинамические свойства индивидуальных соединений / Под ред. В. ІІ. Глушко. - М.: Наука, 1982. - T. Barin, I. Thermochemical Data of Pure Substances / I. Barin // Third Edition. New York: VCH Publishers, Inc., 1995. - 2003 p.

14. Chase, M. W. Themochemical Tables, / M. W. Chase // Journal of Physics and Chemistry Reference Data. - 4th edition, 1998. - Monograph 9. - P. 1-19.

15. Binnewies, M. Thermocliemical Data of Elements and Compounds /M.Binnewies, E. Milke.Weinheim:Wiley-VCH Verlag GmbH, 2002. - 928 p.

16. Binary Alloy Phase Diagrams / Ed. by T.B. Massalski [etc.], Metals Park: ASM International, 1990. - $1824 \mathrm{p}$.

17. Диаграммы состояния двойных металлических систем: справочник / Под ред. Н. П. Лякишева. - М: Машиностроение, 1999. - Т. 2. - 1023 с.

18. Двойные и многокомпонентные системы на основе меди : справочник / М. Е. Дриц [и др.]. - М: Наука, 1979. - 248 с.

19. Sereda B. Increased Strength Multi-phase Steels as a Result of Heat Treatment of Automobile Wheels/ Sereda D., Sereda B. // Material science and technology-2016. Salt Lake City, Utah USA, $2016-1339$ p. - P. 1055-1060

20. Sereda B. Obtaining of Boride Coatings under SHS Conditions for Car Parts / Sereda D., Sereda B. // Material science and technology-2016. Salt Lake City, Utah USA, 2016 - 1339 p. - P. 945-948.

\section{THERMODYNAMIC MODELING OF THE PRODUCTION OF TITANIUM DOPED ALUMINIZED COATINGS UNDER CONDITIONS OF SELF-PROPAGATING HIGH-TEMPERATURE SYNTHESIS Sereda B.P., Gaidaenko A.S., Sereda D.B.}

The study of the formation of a protective coating on structural materials was carried out using the method of thermodynamic analysis of possible chemical reactions between the components of the system. For this purpose, the interaction of the gas saturable medium formed in the process of SHS and material was calculated. For the thermodynamic analysis of the SHS process for the formation of aluminized coatings, a universal program for calculating multicomponent heterogeneous TERRA systems, created on the basis of the ASTRA-4 program for the WINDOWS environment, has been developed for high-temperature processes. Unlike traditional methods in chemical thermodynamics for calculating equilibrium parameters using Gibbs energy, equilibrium constants and the law of effective mass of Goldberg and Vahe, the universal program of thermodynamic calculations TERRA is based on the principle of maximum entropy for isolated thermodynamic systems in equilibrium. It is characterized by the maximum entropy in thermodynamic degrees of freedom, which include the concentration of system components, temperature, pressure.

With increasing temperature, the amount of products in the gaseous phase increases and condensed products are released. It is characteristic that in the temperature range of 700-1600 K the fraction of the condensed phase decreases, which is associated with the evaporation of the carrier. At the same time, starting from a temperature of $800 \mathrm{~K}$, the decomposition of the reaction products occurs, confirms the appearance of decomposition products and a sharp increase in the number of moles of gas. Gaseous products interact with the elements of the powder system is transferred to the gas phase. At temperatures above $800 \mathrm{~K}$, the fraction of the condensed phase remains almost unchanged. This fact suggests that in the temperature range $700-1600 \mathrm{~K}$ reactions occur with the release of the condensed phase, but without changing the number of moles, which is typical of decomposition reactions, exchange with the lining, that is, in fact there is a chemical transport of elements. 
The content of gaseous aluminum compounds in the reactor in the thermal self-ignition mode of the SHS charge for the system: $25 \%$ cholesterol $+15 \% \mathrm{Al}+15 \% \mathrm{Ti}+40 \% \mathrm{Al} 2 \mathrm{O} 3+2 \% \mathrm{NH} 4 \mathrm{I}+$ 3AlF3. In the temperature range of 700-1600 K, there are: $\mathrm{AlH}, \mathrm{AlH} 2, \mathrm{AlH} 3, \mathrm{AlF}, \mathrm{AlF} 2, \mathrm{AlF} 3, \mathrm{AlHF}$, AlH2F, AlI, AlI2, AlI3, etc. The content of gaseous chromium compounds: Al (c), Al2O3 (c), AlF3 ( c), All3 (c), AlN (c), Cr (c), Cr2 (c), CrI (c), Ti (c), etc. The content of gaseous titanium compounds: TiOH, TiF, TiF2, TiF3, TiF4, TiOF2, TiI, TiI2, TiI3, TiI4, TiOF, etc. As well as the content of condensed products: $\mathrm{Al}$ (c), $\mathrm{Al} 2 \mathrm{O} 3$ (c), $\mathrm{AlF} 3$ (c), $\mathrm{AlI} 3$ (c), $\mathrm{AlN}$ (c), $\mathrm{Cr}$ (c), $\mathrm{Cr} 2$ (c), CrI (c), Ti (c), etc.

As a result of the thermodynamic calculation of the equilibrium composition of the reaction products, kinematic schemes of chemical transformations in the systems under study are obtained. Condensed and gaseous compounds are determined. It was proved that in the temperature range of $700-1600 \mathrm{~K}$ reactions occur with the release of the condensed phase, but without changing the number of moles, which is typical of decomposition reactions, exchange with structural material, that is, chemical transport of elements occurs.

\section{References}

[1] Termodinamicheskie svojstva individual'nyh veshchestv: Spravochnoe izdanie v 4-h t. / L. V. Gurvich, I. V. Vejc, V. A. Medvedev i dr.- M: Nauka, 1978.

[2] JANAF Thermochemical Tables (Third Edition). J.Phys. Chem. Ref. Data vol.14 (1985) Suppl. No.1. (см. также NIST Chemistry WebBook: http://webbook.nist.gov).

[3] Edil'baev A.I. / Issledovanie vozmozhnosti obezzhelezivaniya margancevyh koncentratov / Vestnik Nacional'noj inzhenernoj akademii RK Almaty., 2008. - № 4. - S. 125-133.

[4] Primenenie EHVM dlya termodinamicheskih raschetov metallurgicheskih processov / [ Sinyarev G. B., Vatolin N. A.,Trusov B. G., Moiseev R. K. i dr.]. - M.: Nauka, 1982. - 263 s.

[5] Zmij V.I. Osobennosti vakuumnogo aktivirovannogo diffuzionnogo nasyshcheniya metallov: termodinamika, mekhanizm i kinetika / V.I. Zmij, S.G. Ruden'kij // Metallofizika i novejshie tekhnologii., 1998. - № 10. - S. 69-75.

[6] Lovshenko F. G. Termodinamicheskoe modelirovanie geterogennogo vzaimodejstviya pri mekhanicheskom legirovanii v sistemah na osnove medi / F. G Lovshenko, G. F. Lovshenko, B. B. Hina, 3. M. Lovshenko, I. A. Lozikov//Vestnik Belorussko-Rossijskogo universiteta., 2012.№ 1(34). - S. 23-35.

[7] Primenenie EHVM dlya termodinamicheskih raschetov metallurgicheskih processov / G. B. Sinya-r v [i dr.]. - M.: Nauka, 1982. - $264 \mathrm{~s}$.

[8] Mason, P. Modeling thermodynamic processes / P. Mason, M. Mignanelli // Advanced Materialsand Processes., 1998. - Vol. 153, №. 4. - P. 21-24.

[9] Shiryaev, A. Thermodynamics of SIIS processes: An advanced approach / A. Shiryaev // International Journal of'SHS., 1995. - № 4. - P . 351-362.

[10] Barin, I. Thermoeheinical Properties of Inorganic Substances. Supplement / I. Barin, O. Knacke, 0. Kubaschevski. - Berlin: Springer-Verlag, 1977. - 243 p.

[11] Termodinamicheskie svojstva neorganicheskih, veshchestv: spravochnik / U.D. Veryatin [i dr.]. : Atomizdat, $1965 .-460 \mathrm{~s}$.

[12] Termicheskie konstanty veshchestv / Pod red. V.II. Glushko M. VINITI, 1979 - 197 s.

[13] Termodinamicheskie svojstva individual'nyh soedinenij / Pod red. V. II. Glushko. - M.: Nauka, 1982. - T. Barin, I. Thermochemical Data of Pure Substances / I. Barin // Third Edition. - New York: VCH Publishers, Inc., 1995. - 2003 p.

[14] Chase, M. W. Themochemical Tables, / M. W. Chase // Journal of Physics and Chemistry Reference Data. - 4th edition.,1998. - Monograph 9. - P. 1-19.

[15] Binnewies, M. Thermocliemical Data of Elements and Compounds /M. Binnewies, E. Milke.Weinheim:Wiley-VCH Verlag GmbH, 2002. - 928 p.

[16] Binary Alloy Phase Diagrams / Ed. by T. B.Massalski [etc.], - Metals Park: ASM International, 1990. $-1824 \mathrm{p}$.

[17] Diagrammy sostoyaniya dvojnyh metallicheskih sistem: spravochnik / Pod red. N. P. Lyakisheva. - M: Mashinostroenie, 1999. - T. 2. - $1023 \mathrm{~s}$. 
[18] Dvojnye i mnogokomponentnye sistemy na osnove medi: spravochnik / M. E. Dric [i dr.]. - M: Nauka, 1979. $-248 \mathrm{~s}$.

[19] Sereda B. Increased Strength Multi-phase Steels as a Result of Heat Treatment of Automobile Wheels/ Sereda D., Sereda B. // Material science and technology-2016. Salt Lake City, Utah USA 2016 - 1339p. - P. 1055-1060

[20] Sereda B. Obtaining of Boride Coatings under SHS Conditions for Car Parts / Sereda D., Sereda B. //Material science and technology-2016. Salt Lake City, Utah USA 2016 - 1339 p. - P. 945-948. 